\title{
Ontogeny of behavioral arousal in the mouse: Effect of prior testing upon age of peak activity
}

\author{
Z. MICHAEL NAGY and MICHAEL RITTER \\ Bowling Green State University, Bowling Green, Ohio 49403
}

\begin{abstract}
Postnatal locomotor activity was measured in mice as a function of age and repeated test sessions in order to determine whether prior testing would affect the age at which peak activity occurred. Consistent with previous data gathered with single test sessions, peak activity was indicated at 13-14 days of age in two experiments. These findings suggest that behavioral inhibitory capacities mature earlier in the mouse than in the rat. As some evidence of inhibitory functioning was noted several days earlier than peak activity occurred, these results support the hypothesis that the activity peak reflects a transitional stage of brain development from primarily brainstem excitatory functioning to primarily forebrain inhibitory functioning.
\end{abstract}

Recent studies have clearly established that spontaneous activity exhibited postnatally by rats forms an inverted U-shaped function with increasing age, with peak activity occurring between 16-19 days of age (Moorcroft, Lytle, \& Campbell, 1971). Campbell and his colleagues have suggested that these activity changes with age may reflect a caudal-rostral sequence of development of central nervous system (CNS) structures, in which brainstem excitatory centers mature earlier during ontogeny than do telencephalic inhibitory centers, and they have provided evidence through pharmacological experiments in support of this hypothesis (Campbell, Lytle, \& Fibiger, 1969; Campbell \& Mabry, 1973; Mabry \& Campbell, 1974).

A second line of evidence in support of Campbell's hypothesis derives from cross-species comparisons of postnatal activity in species known to differ in stage of CNS maturation at birth. The guinea pig, which is born at a relatively advanced stage of neural maturation, does not show a postnatal activity peak (Campbell \& Mabry, 1972; Oakley \& Plotkin, 1975), while the rabbit, which is roughly intermediate to the rat and guinea pig in terms of neural maturation at birth, exhibits an activity peak at 5 days of age (Oakley \& Plotkin, 1975).

Nagy, Murphy, and Ray (1975) have recently reported that the mouse exhibits a relationship between spontaneous activity and age which is similar to the rat, except that peak activity occurs at 13 days of age. While this finding is in general agreement with evidence that postnatal CNS maturational processes occur slightly earlier in the mouse than the rat (cf. Agrawal \& Himwich, 1970; Folch, Casals, Pope, Meath, LeBaron,

This research was supported by grants from the National Science Foundation (GB-30456) and the National Institute of Child Health and Human Development (HD-09145-01) to the senior author. Requests for reprints should be sent to Z. Michael Nagy, Department of Psychology, Bowling Green State University, Bowling Green, Ohio 43403.
\& Lees, 1959), the age at which peak activity occurred in the mouse was somewhat later than might be predicted from learning studies using neonatal and infant mice. For example, Nagy, Misanin, Newman, Olsen, and Hinderliter (1972) have reported that infant mice between 9 and 10 days of age are capable of learning to suppress a pivoting response in an escape training paradigm and of maintaining a low level of pivoting for at least $24 \mathrm{~h}$ following training. As yoked control groups did not show a similar retention of suppression, Nagy et al. (1972) concluded that the suppression demonstrated by the trained groups represented a learned inhibition of the pivoting response. Therefore, if the activity peak reported by Nagy et al. (1975) does represent the onset of telencephalic inhibitory functioning at 13 days of age, it would be difficult to attribute the inhibitory learning at earlier ages to forebrain functioning.

Moorcroft et al. (1971), however, reported two different ages at which peak activity occurred in the rat, depending upon whether subjects had prior test experience or not. When separate groups were tested at each age for a 23-h session, peak activity occurred at 19 days of age. However, when a single group of rats was tested on consecutive daily 4-h sessions from 0 to 28 days of age, peak activity was indicated at 16 days of age. As the Nagy et al. (1975) study employed separate groups tested at each age for a 4-h session, it is possible that repeated testing of a single group of mice would have resulted in peak activity occurring at an earlier age. Therefore, the purpose of the present study was to determine whether prior testing would result in peak activity occurring earlier than 13 days of age in mice.

\section{EXPERIMENT I}

\section{Method}

Subjects. The subjects were 180 Swiss-Webster mice (Mus musculus), half of each gender, born and reared in 
$30.4 \times 18 \times 12.8 \mathrm{~cm}$ polyethylene cages with wire-grid tops, wood-chip shavings on the floor, and nesting material provided. Litters were culled to eight pups the day following birth and, except for the testing sessions, the mothers remained with the pups through 33 days of age, with food and water available ad lib. The subjects were between 5 and 29 or 100 days of age at the beginning of testing. The 100-day-old mice were littermates of younger mice, were weaned at 33 days of age, and were then maintained in groups of five like-sexed mice until testing. The colony room was maintained at $24 \pm 1^{\circ} \mathrm{C}$ and was on a normal $12-\mathrm{h}$ light-dark cycle.

Apparatus. Three sizes of Plexiglas activity monitoring cages were used. Mice between 5 and 17 days of age were tested in $15.4 \times 5.2 \times 8 \mathrm{~cm}$ cages (small), with red-filtered light sources and photocells placed $2.8 \mathrm{~cm}$ from each end. Mice between 13 and 33 days of age were tested in $19.4 \times 6.4 \times 9 \mathrm{~cm}$ cages (medium), with photocells placed $3.4 \mathrm{~cm}$ from each end, while mice between 29 and 33 and 100 and 104 days of age were tested in $26.2 \times 7.6 \times 10 \mathrm{~cm}$ cages (large), with photocells placed $5 \mathrm{~cm}$ from each end. The floors of the small and medium cages were composed of 1-mm-diam stainless steel rods spaced $4 \mathrm{~mm}$ center to center and extending parallel to the length of the cage, while the floors of the large cages were composed of 1 -mm-diam stainless steel rods $6 \mathrm{~cm}$ center to center and running parallel to the width of the cages.

All of the activity cages were enclosed in a temperatureand humidity-controlled environmental chamber (24 $\pm 1{ }^{\circ} \mathrm{C}, 50 \%$ humidity) in which a ventilation fan furnished a 74-dB noise level and two 60-W fluorescent ceiling lights provided the illumination.

Activity counts were registered when the subject crossed alternate photocells, wired in such a manner so as to require the mouse to at least traverse the distance between photocells in order to register a single count. Total activity counts were recorded on apparatus located outside the soundproofed environmental chamber.

Procedure. Subjects were tested for five consecutive daily 2-h sessions spaced $24 \mathrm{~h}$ apart beginning at 5, 9, and 13 days of age in the small cages; $13,17,21,25$, and 29 days of age in the medium cages; and 29 and 100 days of age in the large cages. The separate groups were comprised of nine males and nine females according to a modified split-litter design so that no more than one male and one female from the same litter were assigned to the same group.

At the appropriate age, subjects were removed from the home cage and placed into the activity cage for the 2-h session, after which they were replaced in the home cage. Activity cages were cleaned and checked between each session. All sessions were conducted between 0900 and $1600 \mathrm{~h}$ during the normal 12-h light cycle. The mice were not disturbed or handled other than during the testing sessions and for normal cage maintenance.

\section{Results}

The mean numbers of cage crossings for the $2-\mathrm{h}$ sessions are presented in Figure 1 as a function of age group, test day, and cage size. A three-way analysis of variance with one repeated measure was conducted on these data (Groups 13-17 medium cage and 29-33 large cage were excluded from this analysis), with the factors being age group, sex, and test day. As suggested by the figure, the effects of age group $[F(7,128)=19.88$, $\mathrm{p}<.0005]$, test day $[\mathrm{F}(4,512)=13.42, \mathrm{p}<.0005]$, and the interaction of Age Group by Test Day $[F(28,512)=17.52, p<.0005]$ were highly significant.

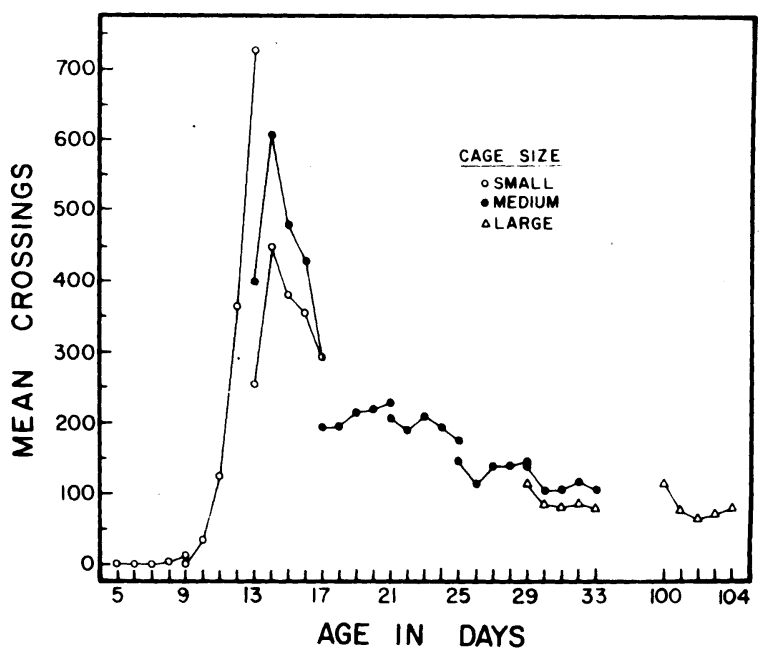

Figure 1. Mean number of daily crossings during 2-h sessions over 5 consecutive days as a function of age at testing and size of test apparatus.

There were no differences due to gender $[F(1,128)=.06]$. Duncan's multiple range tests, conducted across days within each age group, indicated a significant increase in crossings from the first to last day by the $9-13$ group, and a reliable increase from 13 to 14 and then decrease from 14 to 17 days of age by the 13-17 day group (all ps $<.05$ ). The remaining groups failed to show reliable changes across test days. Additional comparisons indicated that activity levels decreased to adult levels by 25 days of age.

In order to determine whether the change in cage size had any appreciable effect upon activity levels, separate analyses of variance were conducted on the groups tested in the small and medium cages at 13-17 days of age and on the groups tested in the medium and large cages at 29.33 days of age. Neither analysis indicated a reliable difference due to cage size.

Finally, $\mathrm{t}$ tests were conducted among the activity scores for the last test day of the 9.13 group and the first test day for the 13-17 groups to determine whether prior testing had an effect upon activity. The group beginning testing at 9 days of age was significantly more active at 13 days of age than either group beginning testing on that day $[\operatorname{ts}(34)>2.76$, ps $<.01]$, while the two groups beginning testing at 13 days of age did not differ reliably from each other $[\mathrm{t}(34)=1.41]$.

\section{EXPERIMENT II}

The results of Experiment I suggest that young mice show an increase in spontaneous activity until 14 days of age, followed by a rapid decrease to adult levels by 25 days of age. However, although previous test sessions appeared to result in higher activity levels at age 13 for the group tested between 9 and 13 days 
of age, it was not possible to determine whether prior testing resulted in an earlier or later peak in activity as compared to groups beginning testing at 13 days of age. The following experiment addressed this question.

\section{Method}

Subjects. Fifty-four mice, born and reared as described in Experiment I, were assigned to one of three groups, each composed of nine males and nine females.

Apparatus. The medium cages described in Experiment I were used.

Procedure. Beginning at 9,11 , or 13 days of age, each group was tested for daily 2-h sessions through 17 days of age as described in Experiment $\mathrm{I}$.

\section{Results}

The mean numbers of cage crossings for the $2-h$ sessions are presented in Figure 2 as a function of test days and age group. Separate analyses of variance conducted upon the activity scores over test days for each of the groups indicated significant increases and then decreases in activity for each group (all ps $<.05$ ). It is also obvious that the group beginning testing at 9 days of age reached peak activity 1 day earlier than groups beginning at either 11 or 13 days of age. While prior testing resulted in the $9-17$ day group being more active at 11 days of age than the 11-17 day group $[\mathrm{t}(34)=2.64, \mathrm{p}<.02]$ and more active than both the $11-17$ and 13-17 day groups at 13 days of age $[\operatorname{ts}(34)>2.62$, ps $<.02]$, the prior testing effect was not a general one, as prior testing did not result in the 11-17 day group having higher activity levels than the 13-17 day group at 13 or even 14 days of age $[\operatorname{ts}(34)<1.0]$.

\section{DISCUSSION}

The results of the present experiments clearly indicate that the use of a single group of mice tested over repeated days for

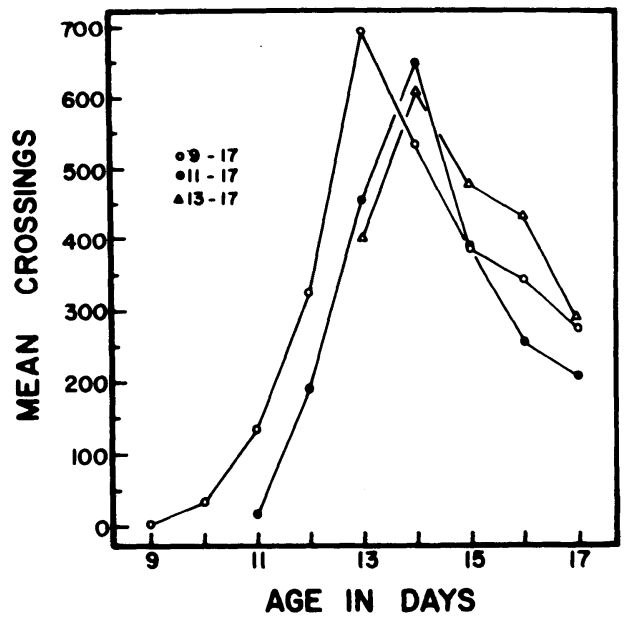

Figure 2. Mean number of daily crossings during 2-h sessions over consecutive days as a function of age at initiation of testing. 2-h sessions does not significantly alter the relationship between age and spontaneous locomotor activity reported in an earlier experiment (Nagy et al., 1975) where separate groups were tested at each age for a single 4-h session. In both instances, an inverted $U$-shaped function was obtained, with peak activity at 13-14 days of age. Assuming that the mice used in the present study and that of Nagy et al. (1975) were equal in terms of CNS maturation at equivalent chronological ages, the failure to find differences in the age at which peak activity occurred would be expected if that activity peak depended primarily upon maturational processes in the CNS.

While there are many procedural differences between the present experiments and those of Moorcroft et al. (1971), it is possible that Moorcroft et al. found an earlier peak with rats tested over repeated days because their groups were not equivalent in terms of CNS maturation. The groups which were tested over repeated days began testing on the day of birth and continued through 28 days of age. It is possible that the repeated handling during the first week or so resulted in an earlier maturation of CNS processes and, hence, an earlier peak in activity. Schapiro and Vukovich (1969) have demonstrated that extra stimulation during the first week of life results in accelerated development of at least one index of CNS maturation-dendritic spine growth-by 8 days of age in rats. Thus, Moorcroft et al. may have obtained an earlier peak not because of repeated testing or handling per se, but rather because those treatments were administered during an early critical period. Further evidence for this interpretation accrues from the third experiment reported by Moorcroft et al., in which control rats were tested on repeated days from 15 through 20 days of age. In this case, peak activity was obtained at 20 days of age.

An extremely important question arising from the results of the present study concerns the meaning of the activity peak. If the decline in activity following the peak represents the age of onset of telencephalic inhibitory functioning, it would be difficult to attribute the learning effects reported by Nagy et al. (1972) to inhibitory functioning by the forebrain. However, a more parsimonious interpretation of the activity peak is that, rather than representing the onset of inhibitory functioning, it represents a stage of telencephalic development which is significantly mature to counteract and overcome the brainstem excitatory effects. That is, the telencephalon may be capable of exerting some inhibitory influence prior to the age at which peak activity is noted, but not sufficiently to suppress activity until a later age. Our data lend some support to this hypothesis: Mice which began testing at 9 days of age were more active at 11 and 13 days of age than groups beginning testing at those ages, suggesting that some inhibitory processes were functional at a younger age than indicated by the peak activity. Of course, it is also possible that inhibitory effects observed at ages younger than 13 days in the mouse may be originating from areas which mature earlier during ontogeny than does the telencephalon, such as the diencephalon which has been reported to inhibit reflex-like behaviors (Sedlacek, Svehlova, Sedlackova, Marsala, \& Kapras, 1961). Further research will hopefully clarify the origin of inhibitory effects during ontogeny.

\section{REFERENCES}

Agrawal, H. C., \& Himwich, W. A. Amino acids, proteins, and monoamines of developing brain. In W. A. Himwich (Ed.), Developmental neurobiology. Springfield, Ill: Thomas, 1970. Pp. 287-310.

Campbell, B. A., Lytle, L. D., \& Fibiger, H. C. Ontogeny of adrenergic arousal and cholinergic inhibitory mechanisms in the rat. Science, 1969, 166, 637-638. 
CAmpbell, B. A., \& MABry, P. D. Ontogeny of behavioral arousal: A comparative study. Journal of Comparative and Physiological Psychology, 1972, 81, 371-379.

CAMPBEll, B. A., \& MABRY, P. D. The role of catecholamines in behavioral arousal during ontogenesis. Psychopharmacologia, 1973, 31, 253-264.

Folch, J., Casals, J., Pope, A., Meath, J. A., LeBaron, F. N., \& LEES, M. Chemistry of myelin development. In S. R. Korey (Ed.). The biology of myelin. New York: Hoeber, 1959. Pp. 122-137.

Mabry, P. D., \& Campbell, B. A. Ontogeny of serotonergic inhibition of behavioral arousal in the rat. Journal of Comparative and Physiological Psychology, 1974, 86, 193-201.

Moorcroft. W. H., Lytle, L. D., \& CAmpbell, B. A. Ontogeny of starvation-induced behavioral arousal in the rat. Journal of Comparative and Physiological Psychology, 1971, 75. 59-67.

Nagy, Z. M., Misanin, J. R., Newman, J. A., Olsen, P. L., \&
HinderLiter, C. F. Ontogeny of memory in the neonatal mouse. Journal of Comparative and Physiological Psychology, 1972, 81 380-393.

NAGY, Z. M., Murphy, J. M., \& Ray, D. Development of behavioral arousal and inhibition in the Swiss-Webster mouse. Bulletin of the Psychonomic Society, 1975, 6. 146-148.

OAKLEY, D. A.. \& Plotkin, H. C. Ontogeny of spontaneous locomotor activity in rabbit, rat, and guinea pig. Journal of Comparative and Physiological Psychology, 1975, 89, 267-273.

Schapiro, S., \& Vukovich, K. R. Early experience effects upon cortical dendrites: A proposed model for development. Science, 1969, 167, 292-294.

Sedlacek, J., Svehlova, M., Sedlackova, J., Marsala, J., \& KAPRAS, J. New results in the ontogenesis of reflex activity. Plzensky lekarsky Sbornik. 1961. Suppl. 3, 167-173.

(Received for publication November 13, 1975.) 